\title{
Boundary drive induced formation of aggregate condensates in stochastic transport with short-range interactions
}

\author{
Hannes Nagel ${ }^{1(a)}$, Hildegard Meyer-Ortmanns ${ }^{2(b)}$ and Wolfhard Janke ${ }^{1(c)}$ \\ 1 Institut für Theoretische Physik, Universität Leipzig, Postfach 100 920, 04009 Leipzig, Germany \\ $\mathrm{n}^{2}$ School of Engineering and Science, Jacobs University Bremen, P.O. Box 750561, 28725 Bremen, Germany
}

PACS 05.60.-k - Transport processes

PACS 02.50.Ey - Stochastic processes

PACS 05.70.Fh - Phase transitions

\begin{abstract}
We discuss the effects of particle exchange through open boundaries and the induced drive on the phase structure and condensation phenomena of a stochastic transport process with tunable short-range interactions featuring pair-factorized steady states (PFSS) in the closed system. In this model, the steady state of the particle hopping process can be tuned to yield properties from the zero-range process (ZRP) condensation model to those of models with spatially extended condensates. By varying the particle exchange rates as well as the presence of a global drift, we observe a phase transition from a free particle gas to a phase with condensates aggregated to the boundaries. While this transition is similar to previous results for the ZRP, we find that the mechanism is different as the presence of the boundary actually influences the interaction due to the non-zero interaction range.
\end{abstract}

Condensation phenomena are observed in a broad range of physical processes. While they are originally associated with phase transitions of matter from the gas state to some liquid or solid state, they are also closely related to nucleation and coarsening phenomena. Examples of condensation appear in processes such as the formation of ○reath figures 1], Bose-Einstein condensation [2], polymer aggregation [3], but in a wider sense also in more generic systems like networks as the formation of clusters (4) through the accumulation of links on sites.

For many such systems, the involved condensation process can be modeled as a stochastic transport process with a set of particles occupying a number of discrete - sites. With particles representing microscopic to macroscopic objects and appropriate dynamics a wide spectrum of physical processes has been studied. Examples include refs. 2, 4 mentioned above, but also processes such as wealth condensation [5] or traffic flow [6].

The zero-range process (ZRP) with condensation dynamics 7,8$]$ is a well known paradigm of such transport processes. While it has a fully symmetric steady state, above some critical density $\rho_{\mathrm{c}}$ the symmetry breaks spon-

\footnotetext{
(a) E-mail: hannes.nagel@itp.uni-leipzig.de

(b) E-mail: h.ortmanns@jacobs-university.de

(c) E-mail: wolfhard.janke@itp.uni-leipzig.de
}

taneously and a particle condensate emerges at a single site such that the density at the remaining sites stays critical. When short-range interactions are introduced, a similar condensation process can be observed with the main difference, that condensates can be spatially extended 912 . In this work, however, we shall consider condensation not as an effect in the steady state, but as a signature of a boundary induced phase transition. Such transitions can occur in driven systems, where the drive is implemented in terms of the interaction at the boundary of the system [13]. A well known transport process with such a transition is the totally asymmetric simple exclusion process (TASEP) 14,15], where a high-density, a lowdensity and a maximal-current phase exist [16]. For the ZRP, such effects of open boundaries and driven particle exchange have been studied using analytical and numerical methods by Levine et al. [17.

While the interaction of particles with the boundary of the ZRP is merely the injection and removal of particles, the presence of the boundary influences hopping at nearby sites once short-range interactions are introduced. Within this paper, we use the term open boundaries in the sense that the boundary sites only have one interaction bond towards the bulk of the system. While this is a natural choice for an isolated system and consistent with ref. [17], 


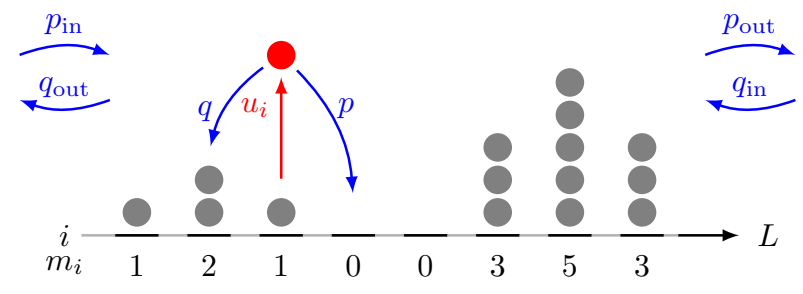

Fig. 1: Schematic representation of the dynamics of a particle hopping process on a one-dimensional lattice with $L$ sites, hopping rate $u_{i}$ and drift parameters $p, q$. At the boundary sites $i=L$ and 1 they are replaced by the removal parameters $p_{\text {out }}$ and $q_{\text {out }}$, respectively. Particle injection (rate parameters $\left.p_{\text {in }}, q_{\text {in }}\right)$ is independent of the hopping rate.

it is also conceivable that the boundary sites interact with a mean-field occupation outside the system, for example to model a compartment of a large system separated by membranes.

We consider a one-dimensional lattice with $L$ sites and a gas of indistinguishable particles. Each site $i$ can hold an arbitrary number $m_{i}$ of particles. Particles can hop between sites as well as enter and exit the system through the boundaries. The dynamics in terms of a discrete stochastic process consists of two steps as indicated in fig. 1. First, a particle may leave from a randomly chosen site $i$ with probability proportional to the hopping rate $u_{i}$. Second, the particle hops to the left with rate $q$ or to the right neighbor with rate $p$. This allows the implementation of symmetric $\left(p=q=1 / 2, p_{\text {in }}=q_{\text {in }}, p_{\text {out }}=q_{\text {out }}\right)$ as well as partially and totally asymmetric $\left(p=1, q=q_{\text {in }}=q_{\text {out }}=\right.$ 0 ) hopping dynamics. At the boundary sites $i=L$ and 1 , the drift parameters $p$ and $q$ are replaced by the removal parameters $p_{\text {out }}$ and $q_{\text {out }}$ which enter into the rates of particle removal $u_{L} p_{\text {out }}$ and $u_{1} q_{\text {out }}$, respectively. The injection rates are independent of the occupation numbers and hence directly given by the parameters $p_{\text {in }}$ and $q_{\text {in. }}$. Due to this explicit particle injection and removal through the open boundaries, the total number of particles $M(t)=\sum_{i=1}^{L} m_{i}$ is not conserved. For the hopping rate function, we consider the form

$$
u_{i}=\prod_{\langle i, j\rangle} \frac{g\left(m_{i}-1, m_{j}\right)}{g\left(m_{i}, m_{j}\right)}
$$

with a symmetric, non-negative weight function $g(m, n)$ given for each bond $\langle i, j\rangle$ of the lattice ${ }^{1}$ Whereas in a closed system with the total number of particles conserved this choice leads to a steady state 9,11 of the form

$$
P_{M, L}(\{m\})=\frac{1}{Z_{M, L}} \prod_{\langle i, j\rangle} g\left(m_{i}, m_{j}\right) \delta_{\sum_{i=1}^{L} m_{i}, M},
$$

this is not necessarily the case with open boundaries. However, since the steady state of the closed system is rooted

\footnotetext{
${ }^{1}$ This form of the hopping rate with fixed $i$ implicitly realizes the fact, that there is no interaction beyond the boundary sites.
}

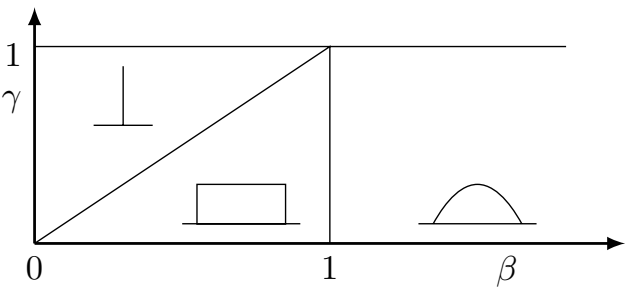

Fig. 2: Regimes of different condensate shapes of the considered tunable model: No condensation for $\gamma>1$, single-site condensates for $\beta<\gamma \leq 1$, rectangular condensates for $\gamma<\beta<1$ and smooth parabolic condensates for $\beta>1$ and $\gamma \leq 1$.

in the factorization property over bonds, i.e., pairs of sites, one usually still refers to it as pair-factorized steady state (PFSS). The normalization constant $Z_{M, L}$ in the steady state (2) has the same function as the partition function in an equilibrium model.

The weight function $g(m, n)$ gives the interaction between particles. Here, we consider the tunable weights

$$
g(m, n)=\exp \left[-|m-n|^{\beta}-\frac{1}{2}\left(m^{\gamma}+n^{\gamma}\right)\right]
$$

proposed by Wacław et al. 10,11. By tuning the parameters $\beta, \gamma$, properties of the condensation process such as the critical density and the condensate's shape and extension can be chosen [12]. This allows us to study the model in a regime with strong nearest-neighbor interactions, where spatially extended condensates occur in the steady state model $(\gamma \leq 1$ and $\gamma<\beta<1$ for rectangular and $\beta>1$ for smooth condensates), while still being able to directly compare to the results of Levine et al. 17] by tuning the model towards the weak nearest-neighbour interaction regime $(\beta<\gamma \leq 1)$, where its properties are similar to the ZRP considered there. Figure 2 summarizes the phases of this model with periodic boundaries.

From the research in ref. 17] we know that the ZRP condensation model with hopping rates $u(m)=1+b / m$ in the condensation regime $b>2$ has a phase transition induced by boundary drive. A homogeneous gas phase where a steady state still exists and one (symmetric hopping) or two (totally asymmetric hopping) phases with single-site condensates aggregated to the boundary are observed. This transition occurs for $p_{\text {in }}>p_{\text {out }}$ for symmetric and $p_{\text {in }}>1$ for totally asymmetric hopping. For the ZRP, the presence of the boundary does not affect the interaction at the boundary sites other than by injection or removal of particles. This makes it possible to understand the condensate formation at a boundary site for totally asymmetric hopping by only considering the difference of the fluxes into and out of that site [17. The occupation number of the boundary site is then treated as a biased bounded random walk with drift equal to this net flux. As shown in fig. 3, this drift becomes positive for sufficiently high influx rate $p_{\text {in }}>1$ and occupation $m_{1}$ so that a condensate can emerge and grow after a sufficiently large 


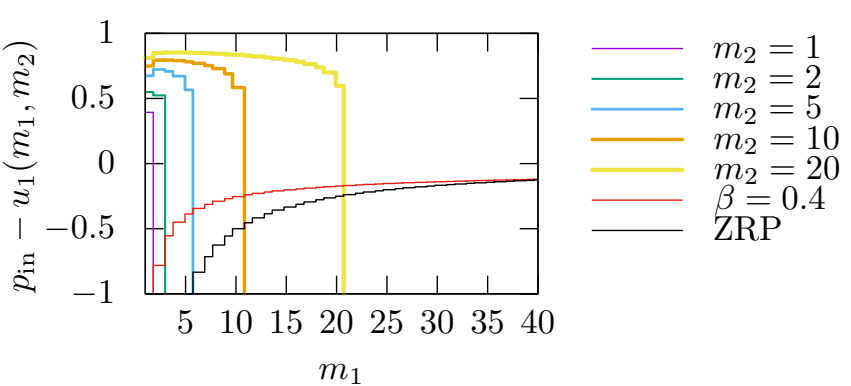

Fig. 3: Drift $p_{\text {in }}-u_{1}\left(m_{1}, m_{2}\right)$ of the occupation number of the first site, $m_{1}$, for different occupations at the second site, $m_{2}$, for our model $(\beta=1.2, \gamma=0.6)$ at $p_{\text {in }}=1$ as well as for weak short-range interactions $\left(\beta=0.4, \gamma=0.6, m_{2}=0\right)$ and the ZRP with totally asymmetric $(p=1, q=0)$ dynamics. For $\beta=0.4$, there is no significant dependence on the second site occupation $m_{2}$, for the ZRP there is none.

fluctuation of $m_{1}$. For the ZRP, the effect of these boundary condensates to the bulk system is then, that they act as particle reservoirs that hold the particle density in the bulk stationary at the critical density $\rho_{\mathrm{c}}=1 /(b-2)$. It is also apparent from fig. 3 that with short-range interactions this drift behaves entirely differently and also depends on the second site's occupation $m_{2}$. We will pick up this observation further below, when we discuss the corresponding phase of aggregate condensate formation for the short-range interaction model.

In our own preliminary work $[18$ we additionally observed for the PFSS model the formation of condensates in the bulk system in the aggregate condensate phase for symmetric hopping. From this we could also assume that with short-range interactions the phase diagram induced by particle exchange and external drive is similar to that of the ZRP. However, there were limitations of the simulation method of the stochastic process because no upper bounds exist for the hopping rate function generated by the weights (3), so that we were not yet able to study the phase diagram with short-range interactions to our satisfaction.

To avoid these problems, we used here an improved rejection-free kinetic Monte Carlo (KMC) method, originally introduced for the simulation of coupled rate equations in chemical systems by Gillespie [19 20]. This allows us to directly use the interaction rates of the system which makes simulation much more efficient in situations where no steady state exists.

In the following we will outline how we identify the phase diagram. As a main observable to indicate the expected phase transition we measure the total number of particles $M(t)$ as shown for symmetric hopping in fig. 4 and estimate the scaling exponent $\alpha$ under the assumption that $M(t) \propto t^{\alpha}$ grows as some power of time due to absorption of a fraction of particles entering the system. We can thus identify regions in $\left(p_{\text {in }}, p_{\text {out }}\right)$, where the system continually absorbs particles and the steady state breaks

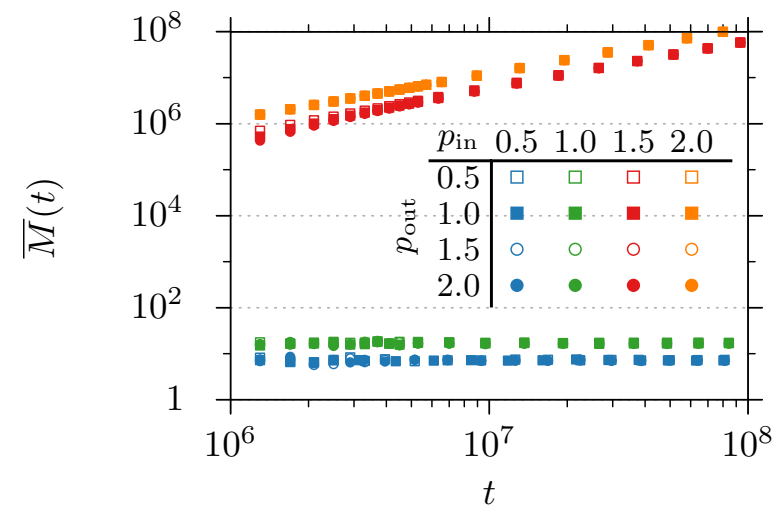

Fig. 4: The total number of particles $M(t)$ in the system versus time. For sufficiently large times, a stationary base line is a signature of the gas phase while linear growth occurs in the aggregate condensate phase. Curves averaged from 25 independent simulations at $\beta=1.2, \gamma=0.6, p=q=1 / 2, L=256$.

down in the regions where $\alpha \approx 1$. This is demonstrated in fig. 5. which shows the average total number of particles versus time for several combinations of particle exchange rates $p_{\text {in }}$ and $p_{\text {out }}$.

In contrast, for sufficiently small values of the influx rate $p_{\text {in }}$ we obtain $\alpha \approx 0$ which reflects that the number of particles in the system remains stationary and suggests that a steady state exists. In the latter case, the particles are homogeneously distributed in the system as a thin gas, rarely interacting (phase $\mathrm{G}$ ). This is visible in the low average occupation number density $\rho_{\text {bulk }}$ in fig. 6 as well as in the average occupation profile shown in fig. 7 (flat profiles). In the gas phase the overall density $\rho$ is identical to the bulk density where any particle accumulations at the boundaries are neglected. In the regions with positive $\alpha$ (dark regions in fig. 5), particles accumulate in the system. This occurs through the emergence of spatially extended condensates at the boundaries, as can be seen in fig. 7. As in this regime the condensates are bound to the boundary, we refer to it as the aggregate condensate phase $(\mathrm{A})$. While for symmetric hopping $(p=q=1 / 2)$ identical formations appear at both boundaries [fig. 7(a)], distinctly shaped and independent condensates are observed for totally asymmetric hopping $(p=1, q=0)$ [fig. $7(\mathrm{~b})]$. These condensates in the latter case appear separately in the regions $A_{\text {in }}$ and $A_{\text {out }}$ or together in the region $A$ of figs. 5 and 6 .

At the boundary with particle influx, for a sufficiently high injection rate $p_{\text {in }}$ in the regime $A_{\text {in }}$, an aggregate condensate forms that obeys the same envelope shape as a bulk condensate in the periodic model [11] [both boundaries in fig. 7(a), left boundary in (b)]. There also appears a regime $A_{\text {out }}$, where particles condense prior to leaving the system, as well as a coexistence region A [for example $p_{\text {in }}=1, p_{\text {out }}=0.2$ in fig. 6(b)]. For symmetric hopping the inbound condensate coexists with the outbound con- 


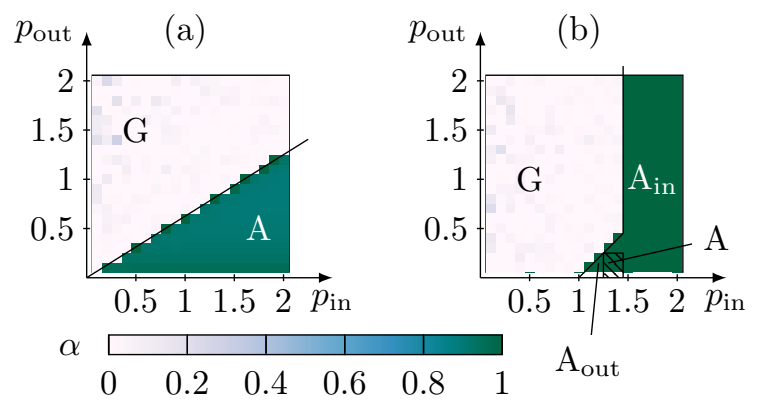

Fig. 5: Scaling exponent $\alpha\left(p_{\text {in }}, p_{\text {out }}\right)$ of the total mass $M(t)$ over time for (a) symmetric $\left(p=q=1 / 2, p_{\text {in }}=q_{\text {in }}, p_{\text {out }}=\right.$ $\left.q_{\text {out }}\right)$ and (b) totally asymmetric $\left(p=1, q=q_{\text {in }}=q_{\text {out }}=0\right)$ dynamics estimated at large times $10^{7}<t<10^{8}$ for $\beta=$ $1.2, \gamma=0.6$.

densate, the former, however, dominates the shape due to its much larger contribution. The transition lines from the gas phase $\mathrm{G}$ to the aggregate condensate phase $\mathrm{A}$ for the coupling constants $\beta=1.2, \gamma=0.6$ are found at $p_{\text {in }}>$ $0.625 p_{\text {out }}$ for symmetric and $p_{\text {in }}>p_{\text {in,crit }}=1.43 \pm 0.02$ for totally asymmetric hopping as shown in figs. 5 and 6 . We also observe that the location of the transition lines depends strongly on the couplings $\beta, \gamma$ as well as the type of interaction at the boundaries. For instance, by decreasing the strength of the short-range interactions, the critical lines approach those observed for the ZRP until they match for $\beta<\gamma$. An interesting modification of the interaction at the boundaries that consists of adding an explicit bond from the boundary sites to an outer site with occupation $m_{\infty}$, effectively introducing fixed boundary conditions, leads to a shift of the transition lines towards larger values of $p_{\text {out }}$ for asymmetric hopping, so that the phases $\mathrm{A}_{\text {out }}$ and $\mathrm{A}$ become significant regions of the phase diagram 21].

While the phase diagrams given in figs. 5 and 6 are similar to those of the ZRP [17, 18, with comparable boundary conditions, we do observe qualitative differences. With short-range interactions, the bulk density of the gas phase increases towards the critical density while approaching the transition line towards aggregate condensate formation (see fig. 6). However, while the occupation density in the bulk system, apart from the aggregate condensates, remains at criticality for the $\mathrm{ZRP}$ as well as weak shortrange interactions $\beta<\gamma$, we observe a sharp decline at the transition line to a bulk density considerably smaller than the critical density here.

On a higher level, with significant short-range interactions (3), i.e. $\beta>1$, the formation of the aggregate condensate at the boundary sites for asymmetric dynamics cannot simply be understood by means of a bounded, biased random walk of the first site's occupation number as with the ZRP. Only with weak nearest-neighbour couplings $\beta<\gamma$ in our considered model this explanation remains valid, as suggested by the monotony and limit

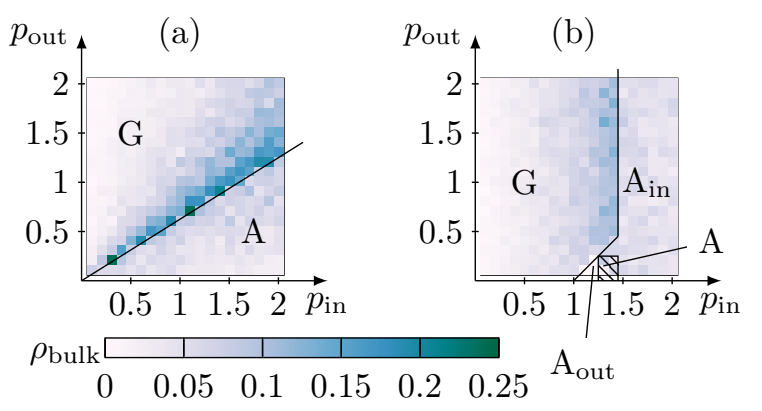

Fig. 6: Bulk density $\rho_{\text {bulk }}\left(p_{\text {in }}, p_{\text {out }}\right)$ for (a) symmetric $(p=$ $\left.q=1 / 2, p_{\text {in }}=q_{\text {in }}, p_{\text {out }}=q_{\text {out }}\right)$ and (b) totally asymmetric $p=$ $1, q=q_{\text {in }}=q_{\text {out }}=0$ ) dynamics with interaction parameters $\beta=1.2, \gamma=0.6$. The critical density observed in a closed system of comparable size $L=M=256$ for these parameters is $\rho_{\text {crit }} \approx 0.3$.

of the drift shown in fig. 3. With short-range interactions such a drift also depends on the occupation of the neighbour site. As shown in fig. 3, for $\beta>1$ this drift is positive even below the observed critical particle influx rate $p_{\text {in }}<p_{\text {in,crit }} \approx 1.43$ for $1 \leq m_{1} \leq m_{2}$ but becomes strongly negative for $m_{1}>m_{2}$. That is, no fluctuation of the first sites occupation alone can lead to growth of the aggregate condensate in this approach. However, the region in which the drift remains positive expands for higher occupations of the second site: The aggregate condensate can only emerge and exist as a spatially extended condensate because a sufficiently high occupation at a couple of the first sites of the system is required to result in positive drift of the first site's occupation number $m_{1}$.

A second mechanism of the emergence of the aggregate condensate is only observed with short-range interactions. For the ZRP or $\beta<\gamma$ the condensate directly forms at the boundary site. With strong short-range interactions $\beta>1$, however, the condensate may initially emerge in the bulk system and then relatively fast connect to the boundary.

We observe boundary drive induced phase separation that becomes visible as the formation of aggregated condensates at the system boundaries from a homogeneous gas phase. The phase diagram itself is similar to that of the ZRP as discussed in ref. [17], specifically the properties of the homogeneous gas phase where the steady state is not broken are much alike. The observed aggregate condensates are spatially extended as expected. The accumulation of particles in these condensates is much stronger than in the ZRP or than expected, decreasing the particle density in the bulk system far below the critical density of the steady state model, therefore excluding the formation of stable droplets in the bulk system.

Since with short-range interactions nearby boundaries do affect the dynamics, it is worthwhile to also look at the influence of the specific implementation of the boundaries. One approach to this could be to assume virtual bonds across the boundaries to a mean-field occupation 
(a)

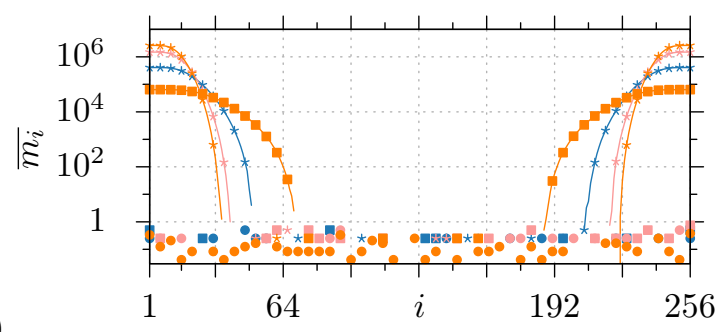

$\left(p_{\text {in }}, p_{\text {out }}\right): \quad *(0.7,0.2) \varpi(0.7,1.2) \bullet(0.7,2.0)$

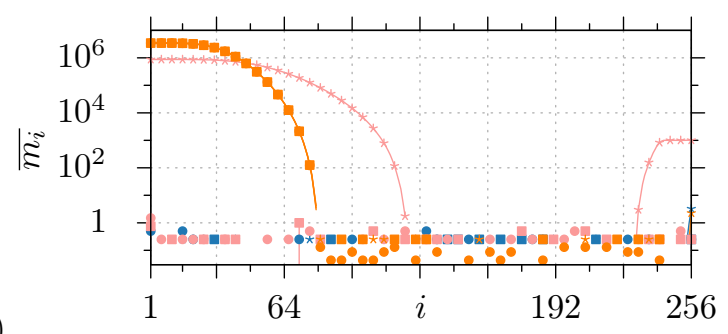

(b)

$$
*(1.0,0.2) \varpi(1.0,1.2) \cdot(1.0,2.0) *(2.0,0.2) \varpi(2.0,1.2) \cdot(2.0,2.0)
$$

Fig. 7: Average occupation number profile for (a) symmetric hopping $\left(p=q=1 / 2, p_{\text {in }}=q_{\text {in }}, p_{\text {out }}=q_{\text {out }}\right)$ and (b) totally asymmetric hopping $\left(p=1, q=q_{\text {in }}=q_{\text {out }}=0\right)$ for varying particle influx and outflux rates. The interaction parameters are $\beta=1.2, \gamma=0.6$ and the system is of size $L=256$.

$m_{\infty}$ as suggested above, so that the homogeneity of the system is not a priori broken by the missing bonds. Although this is a large change of the interaction strengths at the boundary, we merely observe different positions of the phase boundaries as well as a deformation of the shape of the aggregate condensate as it couples to the mean-field occupation $m_{\infty}$ beyond the boundary. A more interesting change is to make particle injection and removal symmetric. With the rate of particle removal set constant to $p_{\text {out }}$, we observe an additional phase with a steady state for symmetric hopping in between the gas and aggregate condensate phases. Most notably, it features a single large condensate that extends the complete bulk of the system but falls to zero nearby the boundaries. For a more detailed discussion of these observations, we refer to ref. 21.

To conclude, we numerically studied the emergence of phase transitions induced by driven particle exchange through open boundaries in a stochastic transport process with tunable short-range interaction. We observed a gas phase and two aggregate condensate phases, where particles accumulate at the boundaries. We presented phase diagrams for the extended smooth condensate regime of the model and discussed the phases' properties. We compared these observations with analytical and numerical results for the zero-range process by Levine et al. 17 where similar phases are observed. While the gas phases in both systems are qualitatively identical, there are noteworthy differences in the aggregate condensate phases such as the spatial extension and in the case of asymmetric hopping different shapes of the aggregate condensates and the below critical bulk density. Furthermore, we found that the mechanism of the formation of aggregate condensates is different as well as supplemented by an additional mechanism, where the condensate forms in the bulk and quickly aggregates at the boundary. Finally, we shortly discussed other types of open boundaries that we deem important for a systematic study of a short-range interaction model with condensation where we observed additional phases featuring a large bulk condensate and a homogeneous fluid, respectively. Therefore, additionally to the boundary drive that our model shares with the ZRP model [17], the spe- cific interaction with the boundary strongly influences the observable phenomena to the point where extra phases become observable.

As an outlook to future work we see many possibilities for further research. For example, when we considered constant removal at the boundary to achieve a symmetry between particle injection and removal, one might additionally take the other choice and use hopping injection, i.e., particles must successfully hop into the system. Another intriguing variation is to actually implement the systems considered here embedded in a larger, possibly periodic, system and interpret the boundaries of the inner system as membranes.

$$
* * *
$$

We would like to thank the DFG (German Science Foundation) for financial support under the twin Grants No. JA 483/27-1 and ME 1332/17-1. We further acknowledge support by the DFH-UFA graduate school under Grant No. CDFA-02-07.

\section{REFERENCES}

[1] Beysens D. and Knobler C. M., Phys. Rev. Lett., 57 (1986) 1433

[2] Bialas P., Burda Z., Petersson B. and Tabaczek J., Nucl. Phys. B, 495 (1996) 463.

[3] Zierenberg J., Mueller M., Schierz P., Marenz M. and Janke W., J. Chem. Phys., 141 (2014) 114908.

[4] Dorogovtsev S.N. and Mendes J.F.F., Evolution of Networks (Oxford University Press, Oxford, 2003).

[5] Burda Z., Johnston D., Jurkiewicz J., Kamiński M., Nowak M. A., Papp G. and Zahed I., Phys. Rev. E, 65 (2002) 2.

[6] Kaupuzs J., Mahnke R. and Harris R. J., Phys. Rev. E, 72 (2005) 056125.

[7] Evans M. R., Braz. J. Phys., 30 (2000) 42.

[8] Evans M. R., Hanney, T., J. Phys. A, 38 (2005) 195

[9] Evans M. R., Hanney T. and Majumdar S. N., Phys. Rev. Lett., 97 (2006) 010602.

[10] Waceaw B., Sopik J., Janke W. and MeyerOrtmanns H., Phys. Rev. Lett., 103 (2009) 80602. 
[11] Waceaw B., SopiK J., Janke W. and MeyerOrtmanns H., J. Stat. Mech.: Theor. Exp., 2009 (2009) P10021.

[12] Ehrenpreis E., Nagel H. and Janke W., J. Phys. A, 47 (2014) 125001.

[13] Popkov V. and Schütz G.M., Europhys. Lett., 48 (1999) 257.

[14] Spitzer F., Adv. Math., 5 (1970) 246.

[15] Blythe R.A., Janke W., Johnston D.A., and Kenna R., J. Stat. Mech.: Theor. Exp., 2004 (2004) P06001.

[16] Krug J., Phys. Rev. Lett., 67 (1991) 1882.

[17] Levine E., Mukamel D. and Schütz G. M., J. Stat. Phys., 120 (2005) 759.

[18] Nagel H., Labavic D., Meyer-Ortmanns H. and Janke W., Phys. Procedia, 57 (2014) 77.

[19] Gillespie D. T., J. Comput. Phys., 22 (1976) 403.

[20] Gillespie D. T., J. Phys. Chem., 81 (1977) 2340.

[21] Nagel H. and Janke, W., in preparation (2015). 